\title{
Correlation analysis of intestinal flora with hypertension
}

\author{
JILUN LIU ${ }^{1 *}$, NING AN $^{2 *}$, CONG MA $^{3}$, XIAOFENG LI ${ }^{4}$, \\ JIE ZHANG ${ }^{5}$, WEI ZHU ${ }^{6}$, YIHE ZHANG ${ }^{7}$ and JUNPENG LI $^{1}$ \\ ${ }^{1}$ Department of Cardiology, No. 215 Hospital of Shaanxi Nuclear Industry, Xianyang, Shaanxi 712000; \\ ${ }^{2}$ Department of Cardiology, Xingyuan Hospital of Yulin City, Yulin, Shaanxi 719000; \\ ${ }^{3}$ Department of Clinical Medicine, Medical College of Nanchang University, Nanchang, Jiangxi 330031; \\ ${ }^{4}$ Department of Clinical Laboratory, Central Hospital of Xi'an City, Xi'an, Shaanxi 710003; \\ ${ }^{5}$ Department of Internal Medicine, No. 5 Hospital of Xi'an City, Xi'an, Shaanxi 710082; \\ ${ }^{6}$ Department of Clinical Laboratory, Central Hospital of Baoji City, Baoji, Shaanxi 721008; \\ ${ }^{7}$ Department of Cardiovascular Medical Center, Dongguan Cardiovascular and Cerebrovascular Disease Hospital, \\ The Affiliated Hospital of Yan'an University, Yan'an, Shaanxi 716000, P.R. China
}

Received August 29, 2017; Accepted June 26, 2018

DOI: $10.3892 /$ etm. 2018.6500

\begin{abstract}
Relationship between intestinal flora content and hypertension was investigated. Ninety-four patients with hypertension who were admitted and treated in No. 215 Hospital of Shaanxi Nuclear Industry from May 2016 to April 2017 were selected as the observation group; and 94 healthy people from the physical examination center of No. 215 Hospital of Shaanxi Nuclear Industry in the same time period were selected as the control group. The systolic (SBP) and diastolic blood pressure (DBP) of all the participants were measured. Reverse transcription-quantitative polymerase chain reaction (RT-qPCR) was used to detect the quantities of Eubacterium rectale, Bacteroides thetaiotaomicron and Bifidobacterium in the intestines, and correlation analyses were performed. The SBP, DBP and content of Eubacterium rectale in the observation group were significantly higher than those in the control group, while the contents of Bacteroides thetaiotaomicron and Bifidobacterium were obviously lower than those in the control group $(\mathrm{P}<0.05)$. Pearson's correlation analysis showed that Eubacterium rectale was positively correlated with SBP and DBP, while Bacteroides thetaiotaomicron and Bifidobacterium had a negative correlation with SBP and DBP $(\mathrm{P}<0.05)$. The results showed that the quantities of Bifidobacterium and Bacteroides thetaiotaomicron are decreased while the number of Eubacterium rectale is
\end{abstract}

Correspondence to: Dr Junpeng Li, Department of Cardiology, No. 215 Hospital of Shaanxi Nuclear Industry, 35 Weiyang West Road, Xianyang, Shaanxi 712000, P.R. China

E-mail: 1i_junpeng249428@163.com

*Contributed equally

Key words: hypertension, intestinal flora, correlation increased in the intestines of patients with hypertension. Moreover, the content of intestinal flora has a significant correlation with hypertension.

\section{Introduction}

With the continuous development of global economy and the accelerated rhythms of life and work, people's daily dietary patterns have changed. As a result, the incidence rate of hypertension is on the increase, and increasingly younger patients are affected by this disease (1). Drug therapies are used as the main way of treatment for hypertension, which consist of $\beta$-receptor blocker, diuretics, calcium channel blocker, angiotensin-converting enzyme inhibitor, angiotensin II receptor blocker and other drugs. Although the therapies can effectively lower the blood pressure and control it at a certain level, they bring a series of side effects to the body at the same time, including abnormal lipid metabolism, evocation of diabetes, increase in heart rate, gastrointestinal dysfunction, abnormal liver function and impairment of renal function. Therefore, it is necessary and important to explore prevention and treatment measures for hypertension $(2,3)$. There are huge numbers of microorganisms in human body, which mainly concentrate in the gastrointestinal tract. Currently the intestinal floras that have been detected are Bacteroides, Firmicutes, Fusobacterium, Actinomycetes, Verrucomicrobia, Proteobacteria, Spirochaetes, Cyanobacteria, VadinBE97 and Methanobrevibacter (4). The intestinal floras are maintained in a relatively balanced state in the human body under normal conditions, which can regulate and nurture the intestines, influence the immune system of the body, effectively prevent the invasion of pathogenic bacteria and have a close association with hypertension (5). Bacteroides thetaiotaomicron is one of the largest microflora in the intestinal tract. Eubacterium rectale is a conditional pathogen. Bifidobacterium is a probiotic bacterium that has a variety of functions in the intestine. The selection of these three bacteria can completely reflect the basic situation of the whole intestinal flora of human 
body. The treatment principle of hypertension is that the blood pressure is decreased slowly and steadily. Therefore, dietary intervention and other non-pharmacologic treatments have a positive effect on the body's metabolism; they have important impacts on the intestinal flora (6). In this study, the content of intestinal flora in hypertension patients was studied, and its correlation with blood pressure was analyzed.

\section{Patients and methods}

General patient data. Ninety-four patients with hypertension who were admitted and treated in No. 215 Hospital of Shaanxi Nuclear Industry (Xianyang, China) from May 2016 to April 2017 were selected as the observation group. Inclusion criteria: i) Patients whose blood pressure was measured 3 times for 2 consecutive days, with systolic blood pressure (SBP) $\geq 140 \mathrm{mmHg}$ and (or) diastolic blood pressure (DBP) $\geq 90 \mathrm{mmHg}$; ii) patients who had clear consciousness and could communicate with others normally; and iii) patients who signed the informed consent. Exclusion criteria: i) Patients with congenital heart disease, severe infectious disease, malignant tumor or serious liver disease; ii) patients with a history of diarrhea or other gastrointestinal diseases in the previous 1 month; iii) patients who took steroidal drugs, antibiotics and probiotics in the prior 4 weeks; and iv) patients with secondary hypertension. Ninety-four healthy people from the physical examination center of No. 215 Hospital of Shaanxi Nuclear Industry in the same time period were selected as the control group. This study was checked and approved by the Ethics Committee of No. 215 Hospital of Shaanxi Nuclear Industry (Xianyang, China), and the differences in the general information between the two groups of participants were not statistically significant $(\mathrm{P}>0.05)$ (Table I) Signed informed consents were obtained from the patients or guardians.

\section{Methods}

Data collection. The background information (demographic characteristics) and disease information (duration of hypertension) of the 94 hypertension patients were collected.

Detection of blood pressure. All the research objects had a rest for $10 \mathrm{~min}$ under appropriate conditions, and then a mercury sphygmomanometer was used to measure the blood pressure of the brachial artery. The blood pressure was measured 3 consecutive times, and the mean value was calculated.

Measurement of intestinal flora. i) Collection of fecal samples: After $2 \mathrm{~g}$ or more fecal sample of each research object was collected and sent to the laboratory, the sample was separated in an Eppendorf (EP) tube and labeled under sterile conditions, and then it was stored at $-80^{\circ} \mathrm{C}$. ii) Collection of cells in the feces: Fecal sample $(0.5 \mathrm{~g})$ was taken and mixed with $10 \mathrm{ml}$ phosphate-buffered saline (PBS) (Beijing Dingguo Changsheng Biotechnology Co., Ltd., Beijing, China); then the sample was centrifuged at $2,750 \times \mathrm{g}$ for $5 \mathrm{~min}$ at $4^{\circ} \mathrm{C}$, the precipitate was discarded and the supernatant was taken; after the procedure was repeated another two times, the supernatant was taken and marked as supernatant I. Supernatant I $(1 \mathrm{ml})$ was added into the EP tube and centrifuged at $10,500 \mathrm{x} \mathrm{g}$ for $10 \mathrm{~min}$ at $4^{\circ} \mathrm{C}$. After the supernatant was discarded, another
Table I. General data of research objects.

\begin{tabular}{|c|c|c|c|c|}
\hline \multirow[b]{2}{*}{ Item } & \multicolumn{2}{|c|}{ Groups } & \multirow[b]{2}{*}{$t / \chi^{2}$} & \multirow[b]{2}{*}{ P-value } \\
\hline & $\begin{array}{l}\text { Observation } \\
\quad(\mathrm{n}=94)\end{array}$ & $\begin{array}{c}\text { Control } \\
(\mathrm{n}=94)\end{array}$ & & \\
\hline Age (years) & $30-78$ & $30-75$ & & \\
\hline $\begin{array}{l}\text { Sex } \\
\text { (male/female) }\end{array}$ & $49 / 45$ & $46 / 48$ & 0.085 & 0.771 \\
\hline $\begin{array}{l}\text { Average age } \\
\text { (years) }\end{array}$ & $57.76 \pm 7.42$ & $58.15 \pm 7.73$ & 0.353 & 0.724 \\
\hline $\begin{array}{l}\text { Body mass index } \\
(\mathrm{BMI})\left(\mathrm{kg} / \mathrm{m}^{2}\right)\end{array}$ & $21.78 \pm 2.54$ & $22.07 \pm 2.23$ & 0.832 & 0.406 \\
\hline \multicolumn{5}{|l|}{$\begin{array}{l}\text { Educational } \\
\text { level }(\mathrm{n}, \%)\end{array}$} \\
\hline $\begin{array}{l}\text { Junior high } \\
\text { school and below }\end{array}$ & $17(18.08)$ & $16(17.02)$ & & \\
\hline $\begin{array}{l}\text { Senior high school } \\
\text { and special } \\
\text { secondary school }\end{array}$ & 46 (48.94) & $49(52.13)$ & 0.192 & 0.909 \\
\hline College and above & $31(32.98)$ & $29(30.85)$ & & \\
\hline Smoking $(\mathrm{n}, \%)$ & $26(27.66)$ & $23(24.47)$ & 0.110 & 0.739 \\
\hline Drinking (n, \%) & 37 (39.36) & 39 (41.49) & 0.022 & 0.882 \\
\hline
\end{tabular}

$1 \mathrm{ml}$ supernatant I was added and centrifuged at $10,500 \mathrm{x} \mathrm{g}$ for $10 \mathrm{~min}$ at $4^{\circ} \mathrm{C}$, and then the supernatant was discarded. After the procedure was repeated twice, the precipitate was taken. iii) Extraction of total DNA of intestinal flora: The total DNAs of the bacteria in the precipitate were extracted strictly according to the instructions of the extraction kit of bacterial genome DNA (Beijing Dingguo Changsheng Biotechnology Co., Ltd.), and ultraviolet spectrophotometric assay was utilized to detect the concentration and purity of the total DNA, so as to ensure that the concentration ratio was 1.6-1.9. iv) Synthesis of primers for reverse transcription-quantitative polymerase chain reaction (RT-qPCR): All the primers were synthesized by Shanghai Sangong Pharmaceutical Co., Ltd. (Shanghai, China). The primers for Eubacterium rectale were: Forward, CATAAAATCATAGGTTCGCATGAA and reverse, TTTTACCCTACCAACCAGCTAATC. The primers for Bacteroides thetaiotaomicron multiforme were: Forward, TGTCGTAACAACGATTTCATAGGT and reverse, TGTTA GACTCGGCTTCTTTCTTTT. The primers for Bifidobacterium were: Forward, TCGCCTCCGGGTGAGAGTGG and reverse, CGAAGCCATGGTGGGCCGTT; v) RT-qPCR: The routine PCR was performed with the genome DNA extracted from the feces in the control group as a template (relevant kits were provided by Beijing Zhongshan Golden Bridge Biotechnology Co., Ltd. (OriGene Technologies, Inc., Rockville, MD, USA). Reaction conditions: Denaturation at $94^{\circ} \mathrm{C}$ for $3 \mathrm{~min}$ and at $94^{\circ} \mathrm{C}$ for $30 \mathrm{sec}$, annealing at $57^{\circ} \mathrm{C}$ for $30 \mathrm{sec}$ and at $72^{\circ} \mathrm{C}$ for $60 \mathrm{sec}$ for 35 cycles, and final elongation at $72^{\circ} \mathrm{C}$ for $5 \mathrm{~min}$. After the PCR underwent electrophoresis, efficient agarose gel DNA recovery kit (centrifugal column type) (Beijing Dingguo Changsheng Biotechnology Co., Ltd.) was used for purification and recovery of DNA which was taken as a standard substance. LightCycler 480 data analysis 
Table II. Comparison of blood pressure and intestinal flora between the two groups.

\begin{tabular}{|c|c|c|c|c|c|c|}
\hline Groups & Case & $\begin{array}{l}\text { Eubacterium } \\
\text { rectale }\left(\times 10^{3}\right)\end{array}$ & $\begin{array}{c}\text { Bacteroides } \\
\text { thetaiotaomicron }\left(\times 10^{3}\right)\end{array}$ & Bifidobacterium $\left(\mathrm{x} 10^{6}\right)$ & $\mathrm{SBP}(\mathrm{mmHg})$ & $\mathrm{DBP}(\mathrm{mmHg})$ \\
\hline Observation & 94 & $3.95 \pm 1.61$ & $2.92 \pm 1.67$ & $2.56 \pm 1.38$ & $145.89 \pm 3.72$ & $94.65 \pm 3.63$ \\
\hline Control & 94 & $2.49 \pm 1.56$ & $4.13 \pm 1.86$ & $9.87 \pm 2.07$ & $129.74 \pm 3.26$ & $85.32 \pm 3.14$ \\
\hline t value & & 6.314 & 4.693 & 28.488 & 31.656 & 18.847 \\
\hline P-value & & $<0.001$ & $<0.001$ & $<0.001$ & $<0.001$ & $<0.001$ \\
\hline
\end{tabular}

SBP, systolic blood pressure; DBP, diastolic blood pressure.

software (Roche Diagnostics, Basel, Switzerland) was applied to draw the standard curves of Eubacterium rectale, Bacteroides thetaiotaomicron and Bifidobacterium; the RT-qPCR was simultaneously performed on blank controls, diluted standard substances and bacterial DNA samples from the feces to be detected [relevant kits were provided by Beijing Zhongshan Golden Bridge Biotechnology Co., Ltd.; OriGene Technologies, Inc.)]. Reaction conditions: $94^{\circ} \mathrm{C}$ for $5 \mathrm{~min}, 94^{\circ} \mathrm{C}$ for $30 \mathrm{sec}, 60^{\circ} \mathrm{C}$ for $30 \mathrm{sec}, 72^{\circ} \mathrm{C}$ for $1 \mathrm{~min}$ for 35 cycles, and $72^{\circ} \mathrm{C}$ for $5 \mathrm{~min}$. The amplification curves were drawn automatically using the instrument for RT-qPCR (PerkinElmer, Inc., Waltham, MA, USA). At the end of the reaction, the copy number of the bacterial genes to be measured was automatically analyzed utilizing the built-in software of the instrument for PCR, which was used as the quantitative result.

\section{Evaluation indexes}

Parameters and indexes of blood pressure. All the patients had a rest for $10 \mathrm{~min}$, and then a mercury sphygmomanometer was used to measure the blood pressure of the upper limbs. The SBP and DBP were measured 3 times, respectively, and the mean values were calculated.

More than $2 \mathrm{~g}$ fecal sample of every research object was collected on the same day, which was separated in an EP tube and labeled, and then it was stored at $-80^{\circ} \mathrm{C}$. RT-qPCR was applied to detect the quantities of Eubacterium rectale, Bacteroides thetaiotaomicron and Bifidobacterium in the intestines.

Statistical analysis. Statistical Product and Service Solutions (SPSS) 19.0 software (IBM Corp., Armonk, NY, USA) was used for data processing. The measurement data were presented as mean \pm standard deviation, and t-test was performed. The enumeration data were presented as ratio and $\chi^{2}$ was used. The Pearson's correlation coefficient was utilized to analyze the correlation. $\mathrm{P}<0.05$ was considered to indicate a statistically significant difference.

\section{Results}

Comparisons of the blood pressure and intestinal flora between the two groups. The comparisons of the blood pressures and intestinal floras between the two groups showed that the content of Eubacterium rectale in the observation group was significantly higher than that in the control group, the differences were significantly significant $(p<0.05)$. And
Table III. Analyses on correlation of SBP and DBP with indexes of intestinal floras.

\begin{tabular}{|c|c|c|c|c|}
\hline \multirow[b]{2}{*}{ Items } & \multicolumn{2}{|c|}{$\begin{array}{c}\text { Correlation } \\
\text { with SBP }\end{array}$} & \multicolumn{2}{|c|}{$\begin{array}{l}\text { Correlation } \\
\text { with DBP }\end{array}$} \\
\hline & $\mathrm{r}$ & $\mathrm{P}$-value & $\mathrm{r}$ & P-value \\
\hline Eubacterium rectale & 0.413 & 0.014 & 0.409 & 0.009 \\
\hline Bacteroides multiforme & -0.407 & 0.008 & -0.405 & 0.006 \\
\hline Bifidobacterium & -0.426 & 0.013 & -0.416 & 0.012 \\
\hline
\end{tabular}

SBP, systolic blood pressure; DBP, diastolic blood pressure.

the levels of SBP and DBP in the observation group were significantly higher than those in the control group, the differences were significantly significant $(\mathrm{p}<0.05)$. While the contents of Bacteroides thetaiotaomicron and Bifidobacterium in the observation group was obviously lower than that in the control group, the differences were significantly significant $(\mathrm{p}<0.05)$ (Table II).

Analyses on correlation of intestinal flora with SBP and DBP. The analyses via Pearson's correlation coefficients demonstrated that the content of Eubacterium rectale was positively correlated with the levels of SBP and DBP, the differences were significantly significant $(\mathrm{p}<0.05)$, and the correlation of the content of Eubacterium rectale with the level of DBP was better than that with the level of SBP. While the content of Bacteroides thetaiotaomicron and Bifidobacterium had a negative correlation with the levels of SBP and DBP, the differences were significantly significant $(\mathrm{p}<0.05)$, and the correlation of the content of Bacteroides multiforme and Bifidobacterium with the level of DBP was better than that with the level of SBP. (Figs 1 and 2; Table III).

\section{Discussion}

Hypertension is a cardiovascular syndrome in a continuous progression status, which can be divided into essential and secondary hypertension (7). The incidence rate of hypertension reaches as high as $20 \%$ or above in some European and American countries. The disease may be triggered by multiple causes, and can lead to functional and structural changes of the heart and blood vessels (8). The pathogenic 

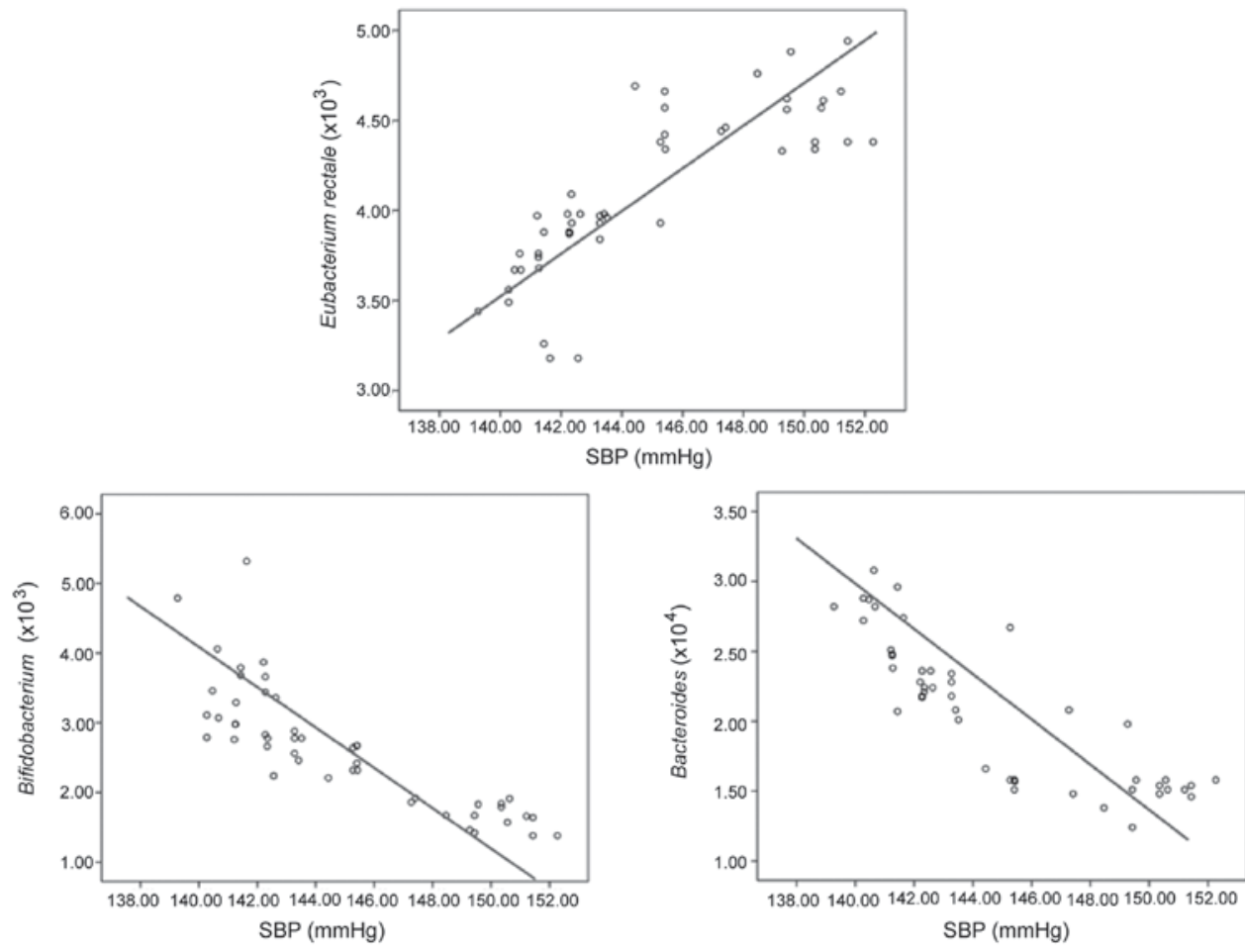

Figure 1. Analyses on correlation of intestinal flora with SBP. The analyses via Pearson's correlation coefficients show that Eubacterium rectale is positively correlated with SBP, while Bacteroides thetaiotaomicron and Bifidobacterium have a negative correlation with SBP $(\mathrm{P}<0.05)$. SBP, systolic blood pressure.
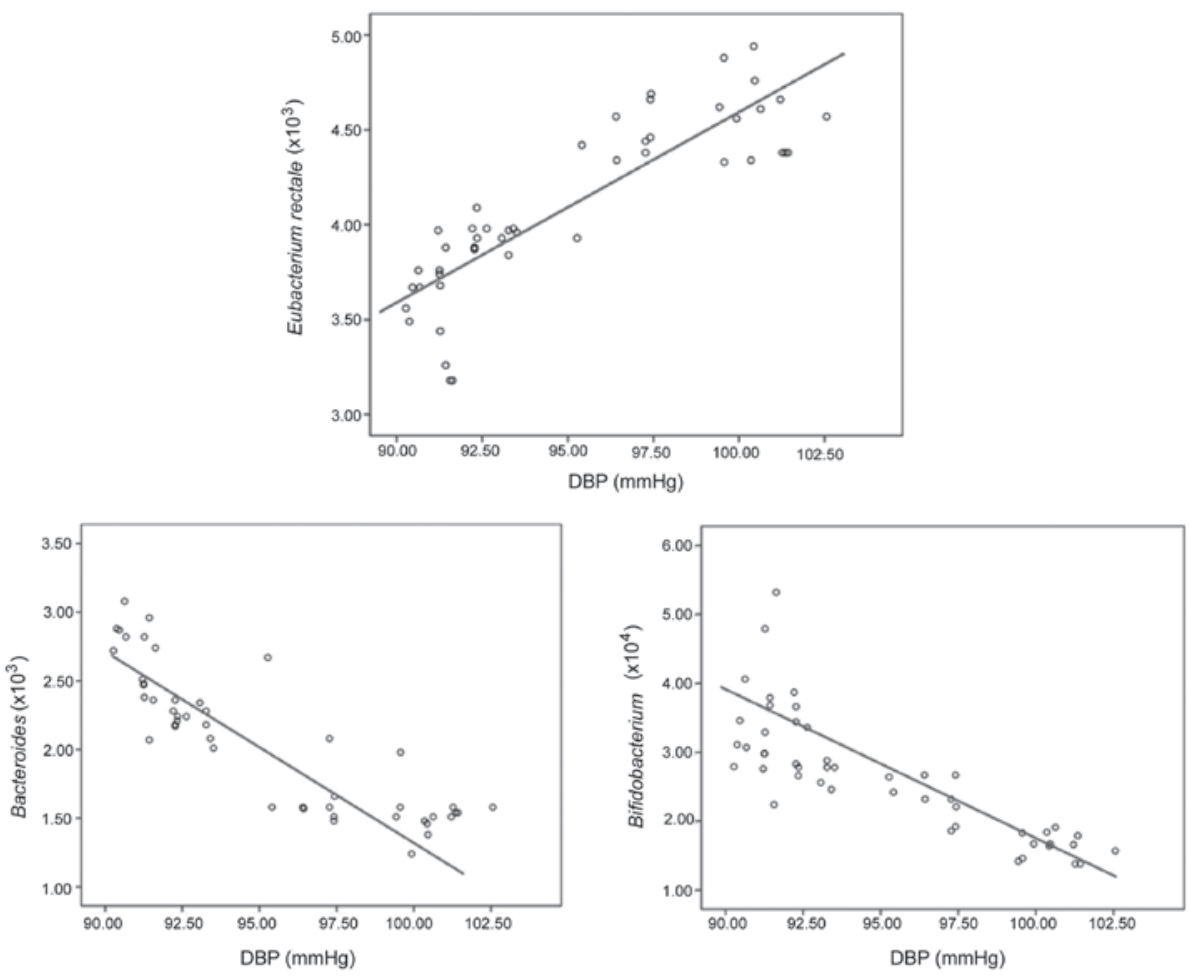

Figure 2. Analyses on correlation of intestinal flora with DBP. The analyses via Pearson's correlation coefficients show that Eubacterium rectale is positively correlated with DBP, while Bacteroides thetaiotaomicron and Bifidobacterium have a negative correlation with DBP $(\mathrm{P}<0.05)$. DBP, diastolic blood pressure.

factors of hypertension are influenced by the environmental and social factors jointly, including toxin effect, infection, diet, medicine, psychological pressure, socioeconomic status and urbanization (9). It is estimated that the intestinal floras in the human body are composed of at least 1,014 bacteria and archaebacteria, which play an important role in human health. The coevolution and symbiosis of the human body and the intestinal floras have formed many physiological functions of the human body, and the intestinal floras are closely related to hypertension. For instance, the blood pressure is generally 
elevated as the quantity of Eubacterium rectale in the intestines is increased (10). The possible mechanism of intestinal floras affecting hypertension is that the intestinal floras can produce short-chain fatty acids (SCFAs) which can regulate the blood pressure through multiple approaches, and control the rising and falling of the blood pressure via orphan $G$ protein-coupled receptor $41(\mathrm{Gpr} 41)$ and olfactory receptor 78 (Olfr78). Among them, Olfr78 can affect the propionate and acetate generated by the intestinal microbiota, thus inducing the release of renin and increasing the blood pressure. Gpr41 can influence the propionate, thus decreasing the blood pressure (11).

Bacteroides thetaiotaomicron is a major representative of Bacteroides and a Gram-negative bacterium. It is also one of the floras and microorganisms that have the largest quantity in the intestines (12). Bacteroides thetaiotaomicron can synthesize vitamins and proteins, so as to help the absorption of food and maintain the balance of the intestinal microecology, playing a dominant role in the intestines (13). Eubacterium rectale is a main representative of Firmicutes and a kind of conditioned pathogen, which can ferment the metabolic products of glucose (such as formic acid, acetic acid and butyric acid) as well as proteins, thereby inhibiting the proliferation of other beneficial bacteria in the intestines and decreasing catabolic enzymes of glycan (14). Bifidobacterium is a class of Gram-positive bacterium, which has multiple functions in the intestines. Therefore, it is a probiotic of great significance for human health (15). The results of this investigation showed that the content of Eubacterium rectale in the observation group was significantly higher than that in the control group, while the contents of Bacteroides thetaiotaomicron and Bifidobacterium were obviously lower than those in the control group $(\mathrm{P}<0.05)$; it may be related to imbalanced structure of intestinal floras, decreased number of probiotics and increased quantity of spoilage bacteria in patients with hypertension.

Some studies have proven (16) that dysbiosis of intestinal floras may have a close correlation with hypertension. The results of this research indicated that Eubacterium rectale was positively correlated with SBP and DBP, while Bacteroides thetaiotaomicron and Bifidobacterium had an inverse correlation with SBP and DBP $(\mathrm{P}<0.05)$, these are consistent with the results of relevant literature which suggest that Eubacterium rectale may be closely related to hypertension. In general, patients with hypertension have a habit of eating food with high fat and protein content. As a result, the harmful bacteria in the intestines are strengthened, especially the reproduction of Eubacterium rectale, thus elevating the blood pressure by promoting vasoconstriction (16). Moreover, a micro-inflammatory state can reduce the diversity of the intestinal floras and accelerate the ecological imbalance of the intestinal floras. Under normal conditions, however, the intestinal floras can work as a defense against the colonization of exotic pathogens, and in turn, the disordered intestinal floras can lead to an overactive state of the body's immune system, thus generating many kinds of inflammatory factors [tumor necrosis factor- $\alpha$ (TNF- $\alpha$ ), interleukin-6 (IL-6) and IL-8], aggravating inflammation and forming vicious cycles (17). Various inflammatory factors can trigger insulin resistance, thus becoming starting factors for hyperten- sion (18). Bacteroides thetaiotaomicron is related to the substance and energy metabolism in the body. When the number of Bacteroides multiforme is decreased, metabolic disorders occur, bringing negative impacts on the metabolic activities, forming metabolic disturbance and inducing hypertension through the pro-inflammatory mechanism (19). Bifidobacterium can suppress the growth of spoilage bacteria and has the actions of adjusting intestinal functions, exerting anti-anaphylaxis and improving nutrition. Therefore, it can have a positive effect on the prevention and treatment of a variety of intestinal diseases (19). Bifidobacterium can synthesize multiple vitamins, promote the absorption of vitamin $\mathrm{D}$ and convert cholesterols into steroids, thus lowering the concentration of cholesterols in the blood and decreasing the blood pressure. When the quantity of Bifidobacterium is reduced, the original balance of the intestinal floras is broken, the release of renin is induced and the blood pressure is elevated (20).

In conclusion, there are abnormal changes in the content of intestinal floras in patients with hypertension. The balance of the whole intestinal floras can be restored through dietary intervention and intake of specific strains and bacterial genera, so as to lower the blood pressure, thus providing new thoughts for onset, prevention and treatment of hypertension. This research adopts a small sample size. Therefore, it is inevitable that there is a bias in the data, and larger sample sizes are needed to further study and prove the results in the future.

\section{Acknowledgements}

Not applicable.

\section{Funding}

No funding was received.

\section{Availability of data and materials}

The datasets used and/or analyzed during the present study are available from the corresponding author on reasonable request.

\section{Authors' contributions}

JiL, NA, CM and XL conceived and designed the study. JiL, JZ, WZ, YZ and JuL were responsible for the collection and analysis of the patient data. NA, CM and JuL interpreted the data and drafted the manuscript. JiL and NA revised the manuscript critically for important intellectual content. All authors have read and approved the final study.

\section{Ethics approval and consent to participate}

The study was approved by the Ethics Committee of No. 215 Hospital of Shaanxi Nuclear Industry (Xianyang, China). Signed informed consents were obtained from the patients or guardians.

\section{Patient consent for publication}

Not applicable. 


\section{Competing interests}

The authors declare that they have no competing interests.

\section{References}

1. Nkeh-Chungag BN, Sekokotla AM, Sewani-Rusike C, Namugowa A and Iputo JE: Prevalence of hypertension and pre-hypertension in 13-17 year old adolescents living in Mthatha - South Africa: A cross-sectional study. Cent Eur J Public Health 23: 59-64, 2015

2. Lin BM, Curhan SG, Wang M, Eavey R, Stankovic KM and Curhan GC: Hypertension, diuretic use, and risk of hearing loss. Am J Med 129: 416-422, 2016.

3. Matsumura K, Arima H, Tominaga M, Ohtsubo T, Sasaguri T, Fujii K, Fukuhara M, Uezono K, Morinaga Y, Ohta Y, et al COMFORT Investigators: Effect of losartan on serum uric acid in hypertension treated with a diuretic: The COMFORT study. Clin Exp Hypertens 37: 192-196, 2015.

4. Wu S, Xu W, Wang FR and Yang XW: Study of the biotransformation of tongmai formula by human intestinal flora and its intestinal permeability across the caco-2 cell monolayer. Molecules 20: 18704-18716, 2015.

5. Yao P, Cui M, Li Y, Deng Y and Wu H: Effects of rhubarb on intestinal flora and toll-like receptors of intestinal mucosa in rats with severe acute pancreatitis. Pancreas 44: 799-804, 2015.

6. Bhatnagar D: Gut flora, diet and intestinal metabolism on cardiovascular risk. Curr Opin Lipidol 26: 148-149, 2015.

7. Pannarale G, Moroni C, Acconcia MC, Pannitteri G, Truscelli G, Valente L, Gentile P, Lopreiato F, Licitra R, Tancredi M, et al: The natural history of prehypertension. A 20-year follow-up. Eur Rev Med Pharmacol Sci 21: 1329-1334, 2017.

8. Hao C, Kang C, Xue J, Shi K, Lv H and Li Z: Effects of blood pressure and sex on heart-vessel coupling in essential hypertension. Turk J Med Sci 46: 680-685, 2016.

9. Ichihara A: Pathogenic mechanism of primary hypertension. Nihon Rinsho 73: 1809-1814, 2015 (In Japanese).

10. Dobrijevic D, Abraham AL, Jamet A, Maguin E and van de Guchte M: Functional comparison of bacteria from the human gut and closely related Non-Gut bacteria reveals the importance of conjugation and a paucity of motility and chemotaxis functions in the gut environment. PLoS One 11: e0159030, 2016.

11. Goffredo M, Mass K, Parks EJ, Wagner DA, McClure EA, Graf J, Savoye M, Pierpont B, Cline G and Santoro N: Role of gut microbiota and short chain fatty acids in modulating energy harvest and fat partitioning in youth. J Clin Endocrinol Metab 101: 4367-4376, 2016.
12. Kasai C, Sugimoto K, Moritani I, Tanaka J, Oya Y, Inoue H, Tameda M, Shiraki K, Ito M, Takei Y, et al: Comparison of human gut microbiota in control subjects and patients with colorectal carcinoma in adenoma: Terminal restriction fragment length polymorphism and next-generation sequencing analyses. Oncol Rep 35: 325-333, 2016.

13. Cuskin F, Lowe EC, Temple MJ, Zhu Y, Cameron EA, Pudlo NA, Porter NT, Urs K, Thompson AJ, Cartmell A, et al: Corrigendum: Human gut Bacteroidetes can utilize yeast mannan through a selfish mechanism. Nature 520: 388, 2015.

14. Rivière A, Gagnon $M$, Weckx S, Roy D and De Vuyst L: Mutual cross-feeding interactions between Bifidobacterium longum subsp. longum NCC2705 and Eubacterium rectale ATCC 33656 explain the bifidogenic and butyrogenic effects of arabinoxylan oligosaccharides. Appl Environ Microbiol 81: 7767-7781, 2015.

15. Milani C, Lugli GA, Duranti S, Turroni F, Mancabelli L, Ferrario C, Mangifesta M, Hevia A, Viappiani A, Scholz M, et al: Bifidobacteria exhibit social behavior through carbohydrate resource sharing in the gut. Sci Rep 5: 15782, 2015.

16. Yang T, Santisteban MM, Rodriguez V, Li E, Ahmari N, Carvajal JM, Zadeh M, Gong M, Qi Y, Zubcevic J, et al: Gut dysbiosis is linked to hypertension. Hypertension 65: 1331-1340, 2015.

17. Peeters AC, Netea MG, Janssen MC, Kullberg BJ, Van der Meer JW and Thien T: Pro-inflammatory cytokines in patients with essential hypertension. Eur J Clin Invest 31: 31-36, 2001.

18. Lai CL, Xing JP, Liu XH, Qi J, Zhao JQ, Ji YR, Yang WX, Yan PJ, Luo CY and Ruan LF: Relationships of inflammatory factors and risk factors with different target organ damage in essential hypertension patients. Chin Med J (Engl) 130: 1296-1302, 2017.

19. Jiang F, Meng D, Weng M, Zhu W, Wu W, Kasper D and Walker WA: The symbiotic bacterial surface factor polysaccharide A on Bacteroides fragilis inhibits IL-1 $\beta$-induced inflammation in human fetal enterocytes via toll receptors 2 and 4. PLoS One 12: e0172738, 2017.

20. Liao ZL, Zeng BH, Wang W, Li GH, Wu F, Wang L, Zhong QP, Wei $\mathrm{H}$ and Fang $\mathrm{X}$ : Impact of the consumption of tea polyphenols on early atherosclerotic lesion formation and intestinal bifidobacteria in High-Fat-Fed ApoE-/- mice. Front Nutr 3: 42, 2016.

This work is licensed under a Creative Commons Attribution-NonCommercial-NoDerivatives 4.0 International (CC BY-NC-ND 4.0) License. 